\title{
The consistency of probabilistic regresses: Some implications for epistemological infinitism *
}

\author{
Frederik S. Herzberg ${ }^{\dagger}$ \\ Copyright notice: The final publication, in ERkEnNTNis 78 (2013), no. 2, pp. \\ 371-382, is available at link.springer.com, doi:10.1007/s10670-011-9358-z
}

\begin{abstract}
This note employs the recently established consistency theorem for infinite regresses of probabilistic justification [F. Herzberg, Studia Logica, $94(3): 331-345,2010]$ to address some of the better-known objections to epistemological infinitism. In addition, another proof for that consistency theorem is given; the new derivation no longer employs nonstandard analysis, but utilises the Daniell-Kolmogorov theorem.

Key words: epistemological infinitism; infinite regress of probabilistic justification; warrant; nonstandard analysis; projective family of probability measures

2010 Mathematics Subject Classification: Primary 00A30, 03A10;
\end{abstract} Secondary 03H05, 60G05

\section{Introduction}

It has been shown recently that infinite regresses of probabilistic justification are 'consistent' in a natural and mathematically rigorous sense (Herzberg [2010]). In the present note, we shall examine how this finding can be employed to defend infinitism even conceptually at various fronts. We shall focus on propositional justification, because the said consistency result primarily pertains to propositional justification and because anyway "doxastic justification is parasitic on propositional justification" (Klein [2007a, 2007b]).

The paper is structured as follows. First (in Section 2), we shall show how the mathematical analysis of infinite regresses of probabilistic justification (pioneered by Peijnenburg [2007]) can serve to illustrate Klein's notion of increasing warrant (i.e. increasing with the number of steps of - in this case: probabilistic - justification). Thereafter, in Section 3 we shall provide, partly on the basis of Herzberg's [2010] consistency theorem, counterexamples to Gillett's [2003] ontological attack on infinitism. Finally, in Section 4. we shall address a possible foundational concern regarding the consistency theorem for probabilistic regresses and its proof. In this connection, we will note the possibility of proving the consistency theorem for infinite regresses

\footnotetext{
${ }^{*}$ It is a pleasure to thank Professor Hannes Leitgeb for helpful conversations.

${ }^{\dagger}$ Institut für Mathematische Wirtschaftsforschung, Universität Bielefeld, Universitätsstraße 25, D-33615 Bielefeld, Germany. E-mail address: fherzberg@uni-bielefeld.de
} 
of probabilistic justification without nonstandard analysis; indeed, Appendix $\mathrm{A}$ provides such a "standard" proof by means of a classical measure-theoretic result that is commonly used for the construction of stochastic processes: the DaniellKolmogorov theorem.

\section{Increasing warrant}

Recall that infinitism agrees with coherentism regarding the progressive and provisional nature of justification:

"[T]he infinitist does not think of propositional justification as a property that is transferred from one proposition to another by such inference rules. Rather, the infinitist, like the coherentist, takes propositional justification to be what I called an emergent property that arises in sets of propositions. In particular, the infinitist holds that propositional justification arises in sets of propositions with an infinite and non-repeating structure such that each new member serves as a reason for the preceding one. Consequently, an infinitist would seek to increase the doxastic justification for an initial belief - the belief requiring reasons - by calling forth more and more reasons. The more imbedded the initial belief, the greater its doxastic justification." (Klein [2007a, p. 26])

This "progress of $[\ldots]$ knowledge" (Klein $[2007 \mathrm{~b}]$ ) - i.e. the progress of propositional and hence also of doxastic justification (the latter being parasitic on the former) - can be illustrated as a process comparable to buying a car on payments (Klein [2007a, p. 28]). A more formal, mathematical illustration of this "progress of $[\ldots]$ knowledge" can be derived from the mathematical analysis of the probabilistic regress problem (cf. Peijnenburg [2007], Peijnenburg and Atkinson [2008] and Herzberg [2010]): For, after $n$ steps of probabilistic justification, one has the following equation for the probability of the proposition $S_{0}$ in question:

$$
P\left(S_{0}\right)=P\left(S_{n+1}\right) \prod_{i=0}^{n} \gamma_{i}+\sum_{\ell=0}^{n} \beta_{\ell} \prod_{i=0}^{\ell-1} \gamma_{i},
$$

wherein for all $k \in \mathbf{N}$

$$
\alpha_{k}=P\left(S_{k} \mid S_{k+1}\right), \quad \beta_{k}=P\left(S_{k} \mid \mathcal{C} S_{k+1}\right), \quad \gamma_{k}=\alpha_{k}-\beta_{k} .
$$

Since $P\left(S_{n+1}\right)$ is unknown and $P\left(S_{n+1}\right) \prod_{i=0}^{n} \gamma_{i}$ may be rather small (unless $\gamma_{i}$ tends to one very rapidly as $i$ goes to infinity, the product diverges to zero, cf. Atkinson and Peijnenburg [2010, Appendix B]), the progress of justification after $n$ steps may be captured in a lower estimate for the probability of $S_{0}$ :

$$
P\left(S_{0}\right) \geq \sum_{\ell=0}^{n} \beta_{\ell} \prod_{i=0}^{\ell-1} \gamma_{i} .
$$

In the limit, one would have

$$
P\left(S_{0}\right)=\lim _{n \rightarrow \infty} P\left(S_{n+1}\right) \prod_{i=0}^{n} \gamma_{i}+\sum_{\ell=0}^{\infty} \beta_{\ell} \prod_{i=0}^{\ell-1} \gamma_{i},
$$


although no infinitist would require that an epistemically responsible agent has to actually complete an infinite regress: to claim that in order for a belief to be justified for someone, that person must have actually completed the process of reasoning to the belief "would be tantamount to rejecting infinitism" (Klein [1998, p. 920]).

(The limit $\sum_{\ell=0}^{\infty} \beta_{\ell} \prod_{i=0}^{\ell-1} \gamma_{i}$ exists, cf. Atkinson and Peijnenburg [2010, Appendix A], whence so does the $\operatorname{limit}_{\lim _{n \rightarrow \infty}} P\left(S_{n+1}\right) \prod_{i=0}^{n} \gamma_{i}=P\left(S_{0}\right)-$ $\sum_{\ell=0}^{\infty} \beta_{\ell} \prod_{i=0}^{\ell-1} \gamma_{i}$.)

This illustration of the progressive and provisional nature of justification can be used to examine two recent critiques of Kleinian infinitism: the one due to Podlaskowski and Smith [2011] as well as Turri's [2009].

Podlaskowski and Smith [2011] admit to be "sympathetic to Klein's suggestion that justification is an ongoing process and that there is 'always a further step that can be taken should we become dissatisfied with the point at which we stopped the progress of inquiry" " (Podlaskowski and Smith [2011, p. 521] citing Klein [2007b]), but they "doubt that the position, as Klein puts it, can be maintained easily" (Podlaskowski and Smith [2011, p. 521]). Their main argument is that on an infinitist account of justification, epistemic agents not only need infinitely many dispositions to form beliefs, but that these have to be given in the right order as well. Hence, they argue that while Klein is justified (if the pun may be excused) in asserting that finite minds may have infinitely many reasons available (Klein [1999, 2007b]), this availability is only of use for a perfectly ordered mind that is capable of producing every reason available when required to do so. Podlaskowski and Smith [2011] doubt that this is an adequate description of the intellectual faculties of ordinary humans, dubbing their argument the finite and less-than-ideally-ordered minds objection (Podlaskowski and Smith [2011, p. 521]). They conclude that an infinitist account of justification would imply that no real human being would ever be able to hold justified beliefs, let alone have knowledge; this result is unacceptable because of the "ought implies can" principle which Podlaskowski and Smith [2011] also subscribe to. A skeptic, who may live with the notion that human knowledge is impossible, could, of course, counter this argument by simply denying this instance of "ought implies can". A non-skeptic infinitist could still maintain that with respect to some questions human minds are ideally ordered and that therefore there are areas where human knowledge is possible even on an infinitist account of justification. In the case of probabilistic justification, there is a third way to respond to Podlaskowski and Smith's argument [2011], viz. by emphasising the progressive and provisional nature of (probabilistic) justification: One might argue that probabilistic justification happens gradually until a sufficient degree of certainty (less than 100\%) has been reached - and above we have given a formal, mathematical description how such an increase of warrant (in the guise of $P\left(S_{0}\right)$ ) looks like. Such a view of probabilistic justification also seems consistent with Klein's view on the subject, who wrote: "As the series lengthens, warrant and credibility increase. Nothing prevents it from increasing to the degree required for knowledge" [2005, p. 138].

Turri [2009, p. 161] writes that even the foundationalist can provide arbitrarily many - indeed, infinitely many! — reasons for a non-evident proposition by inserting intermediate reasons between the foundational belief and the belief in question. Turri conceives of these intermediate reasons as 
being additional pieces in a linear structure of reasons (Turri [2009, figure on p. 160]). First, it must be noted that inserting such intermediate reasons per se does not always increase warrant if these intermediate reaons are fitted into the linear structure of reasons, as Turri suggests. For, suppose $S$ is probabilistically supported by some contingent ${ }^{1}$ event $U$, and suppose that there is another contingent event, $T$, which probabilistically supports $S$ and is itself probabilistically supported by a third contingent event $U$; then, whenever $P(S \Delta T)=0$ or $P(T \Delta U)=02^{2}$ the lower bound in the warrant estimate (2) does not change, even though $S$ and $T$ might not be identical. (It should be noted that unlike the infinitist, the foundationalist cannot move beyond a certain set of "foundational" propositions and thus has to work within the bounds of a linear or at least tree-like structure of reasons.) A defender of Turri's position might respond that such a choice of $S, T, U$ is a knife-edge case, viz. bi-implication; and indeed, such a choice of $S, T, U$ would not be possible in Turri's specific example at the end of his paper [2009, p. 163], because in this example the " $\beta$ 's", viz. $P(S \mid \complement T)$ and $P(T \mid \complement U)$, are positive (cf. Peijnenburg and Atkinson [2011, p. 121]). However, Peijnenburg and Atkinson [2011, Sections 5 and 6] have made a convincing case that the logical structure of Turri's example at the end of his paper actually gives rise to an infinite regress of probabilistic justification - whose consistency follows from the result in Herzberg [2010] or its generalisation in the present paper. Thus they conclude: "Far from supporting Turri's hope that his infinite chain can serve the foundationalist's purpose, the above reasoning has provided ammunition for an infinitist new-style. [...] No anchoring of the infinite sequence of propositions to a basic belief is required. Nor is it possible" (Peijnenburg and Atkinson [2011, p. 122]).

In addition, Turri [2009, p. 161] asserts that before the foundationalist stops, she has also increased warrant. This is true, but by refusing to provide arguments for what she regards as basic beliefs, she makes an ex-ante commitment not to increase warrant as much as she possibly could!

\section{Metaphysical arguments reconsidered}

Gillett's [2003] metaphysical critique echoes a point raised by Fumerton [1998]. Faced with a particularly artificial infinite regress of epistemic justifications, he wrote that "none of them result in any justified beliefs because I don't have any justification for believing the premises of any of the arguments" (Fumerton [1998, p. 936]). Fumerton recognised that there can be infinite regresses that are "metaphysically unproblematic" (Fumerton [1998, p. 936]). Gillett [2003] provides an ontological critique - which he attributes to St Thomas Aquinas of non-repeating epistemic justification in so far as they stipulate the existence of new entities.

Before discussing Gillett's specific argument, two general points about ontological critiques of Kleinian infinitism need to be raised: First, one has to defend Klein against any charge of being short-sighted at this point. Even though he rarely discusses ontological corollaries of epistemological infinitism, Klein does insist that only "epistemically responsible" arguments should count as justifications and prefers at times the term 'warrant' to 'justification'. This

\footnotetext{
${ }^{1}$ An event $U$ is called contingent given a probability measure $P$ if and only if $0<P(U)<1$.

${ }^{2}$ The symmetric difference of two events $A, B$ is defined as $A \Delta B:=(A \backslash B) \cup(B \backslash A)$.
} 
insistence on epistemic responsibility already rules out ontological excesses such as Fumerton's example of a polytheistic regress (where the proposition to be justified is attributed to a certain "god, ${ }_{0}$ " and at the same time it is claimed that " $\operatorname{god}_{n+1}$ " asserts the infallibility of judgments by "god $n$ " for all $n \in \mathbf{N}$ ). The term 'warrant' may also be preferable because it does not carry the same moral connotations that are commonly associated with the term 'justification' (cf. e.g. Podlaskowski and Smith [2011, p. 516] and, for a critique of this widely held view, the article by Fumerton [2001] cited therein). In addition, the term 'warrant' has the advantage of directly taking into account Gettier's insight that justification may be insufficient to turn true belief into knowledge - warrant being usually defined as whatever is needed to turn true belief into knowledge ${ }^{3}$

The second general point about ontological critiques of infinitism is that even a non-repeating regress of epistemic justification which introduces a new entity in each step need not be ontologically extravagant. As we will see presently, whether such a regress becomes epistemically and ontologically irresponsible depends very much on the type of entities and the manner in which they are introduced; consequently, one has to conclude that Gillett's Thomist ontologically motivated rejection of infinitism is mistaken (unless the mathematical actual infinite is denied any status of existence whatsoever).

Gillett [2003] argues against Kleinian infinitism by claiming that Klein must subscribe to (instances of) the following two ontological principles whose combination Gillett finds metaphysically absurd. These ontological principles are (Gillett [2003, p. 712]):

(I) For all entities, an entity $\mathrm{x}$ has a property $\mathrm{H}$ only in virtue of, amongst other possible necessary conditions, some entity y having the property $\mathrm{H}$; and $\mathrm{y}$ is $\mathrm{H}$ only in virtue of some entity $\mathrm{z}$ being $\mathrm{H}$; etc.

(II) For all entities, if entity $\mathrm{x}$ has the property $\mathrm{H}$ only in virtue of, amongst other possible necessary conditions, some entity y having the property $\mathrm{H}$, then $\mathrm{y}$ does not have $\mathrm{H}$ in virtue of $\mathrm{x}$ being $\mathrm{H}$.

In response to this argument, we shall now give two examples of metaphysically unproblematic properties which nevertheless satisfy the two principles (I) and (II) criticised by Gillett. The first example is the property " $E$ is an event that is probabilistically supported by some other event"; this is metaphysically unproblematic in light of the consistency theorem for probabilistic regresses (Herzberg [2010]), as was already noted independently by Peijnenburg [2010]. The second example is the property of being a finite ordinal. One can prove that an ordinal $\alpha$ is finite if and only if there exists some finite ordinal $\beta$ with $\beta>\alpha$. Then, the property of being a finite ordinal satisfies the principles (I) and (II), but since the property of being a finite ordinal can be defined otherwise ${ }^{4}$ it is ontologically unproblematic.

The crux with Gillett's argument is that the phrase "having a property $\mathrm{H}$ in virtue of" is ambiguous. Gillett could choose a more moderate position by only

\footnotetext{
${ }^{3}$ We have nevertheless decided to stick with the term 'justification' for much of this paper simply because it seems to be the more common terminological choice in the literature.

${ }^{4}$ In fact, the property of being a finite ordinal, i.e. a natural number, can even be defined in set-theoretically "robust" terms: It is definable through a $\Delta_{0}$ and thus through an absolute formula. In other words, whether a given set is a natural number does not depend on the interpretation in a particular transitive model of set theory (cf. Jech [2010, Lemma 12.10]).
} 
requiring that any ontologically admissible concept must be definable in some form which avoids the circular structure of principle (I), as it might happen that $\mathrm{H}$ satisfies principles (I) and (II), but can be defined alternatively and therefore is ontologically absolutely harmless. However, this stance no longer presents a categorical opposition to infinitism.

Alternatively, Gillett could reject our counterexamples by adopting an anti-Platonistic view in the philosophy of mathematics. This would mean, however, to commit oneself to a relatively specific stance regarding mathematical ontology, only to exclude a certain option of answering a fundamental epistemological question. Such a philosophical position looks somewhat gerrymandered. One might wonder whether anti-Platonism would be coherent with infinitism of the Kleinian brand. Certainly, Peter Klein's writings — at least those with which the author is familiar — do not contain overtly antiPlatonistic statements; but admittedly, they do not betray an acceptance of mathematical Platonism either.

\section{Addressing foundational reservations against the consistency proof}

As we have seen so far, the consistency of infinite regresses of probabilistic epistemic justification can be used, either directly or by way of illustration, to defend epistemological infinitism against diverse epistemological and ontological objections. Now, the original proof of the consistency of probabilistic regresses uses nonstandard analysis, and it has sometimes been claimed that nonstandard proofs are "not constructive", not even in a broad sense. Recent results from model theory, however, have in fact shown that nonstandard analysis is, in a formal mathematical sense, not "less constructive" than classical analysis (which also employs instances of the Axiom of Choice occasionally, e.g. Zorn's Lemma in the proof of the Hahn-Banach theorem), because in Zermelo-Fraenkel set theory plus Axiom of Choice, there exist definable nonstandard universes (cf. Kanovei and Shelah [2004], Kanovei and Reeken [2004], and Herzberg [2008a, 2008b]). Moreover, whilst nonstandard analysis - from an axiomatic viewpoint a conservative extension of ZFC (e.g. Nelson [1977]) — is of course not intuitionistic, a closer look at the spirit and motivation of concrete applications of nonstandard analysis in mathematics reveals that "[t]he distance between constructive and nonstandard mathematics is [...] actually much smaller than it appears to be" (Schuster, Berger and Osswald [2002, Foreword, p. ix]). And, in any case, one can translate any given nonstandard proof into a "standard" proof by making all the equivalence classes with respect to ultrafilters, which arguments from nonstandard analysis implicitly involve, explicit (usually at the cost of an explosion of the proof's length and complexity).

Nevertheless, an alternative proof — without nonstandard analysis — for the consistency theorem is desirable for purely pragmatic reasons, since not every formal epistemologist can be expected to be familiar with nonstandard methods. Such an alternative, "standard", proof can be found in the Appendix; it employs a technical result from the construction of Markov (i.e. memory-less stochastic) processes, the Daniell-Kolmogorov theorem 5

\footnotetext{
${ }^{5}$ It should be mentioned that the Daniell-Kolmogorov theorem admits a particularly simple
} 
One might ask whether the original, nonstandard proof of the consistency theorem supplies a general proof technique applicable to non-probabilistic settings, too. In other words: Is it possible to construct non-probabilistic examples of non-vicious regresses through a straightforward nonstandard limit construction? The answer is negative: For, suppose $\langle S(n)\rangle_{n \in \mathbf{N}}$ is a sequence of ("standard") mathematical propositions, and assume that there is an infinite regress of non-probabilistic implication, i.e. $\forall n \in \mathbf{N}(S(n+1) \Rightarrow S(n))$. Then, a combination of mathematical induction with the Transfer Principle of nonstandard analysis would yield merely ${ }^{*} S(n+1) \Rightarrow \forall k \leq n\left({ }^{*} S(k)\right)$ for all $n \in{ }^{*} \mathbf{N} 6$ and this formula only carries the regress problem over to a higher level of infinity $\left({ }^{*} \mathbf{N}\right.$ rather than $\left.\mathbf{N}\right)$. Only in the probabilistic setting can a nonstandard limit construction be used to prove consistency of infinite regresses, because in that situation the nonstandard limit can be combined with the Loeb [1975] measure technique of nonstandard probability theory.

\section{Conclusion}

We have seen that the mathematical analysis of infinite regresses of probabilistic justification in general, and the consistency theorem for probabilistic regresses in particular, can be used to fend off several arguments against infinitism, of both epistemological and ontological kind. The consistency theorem and its proof are accessible to anyone with some knowledge of measure-theoretic probability theory and stochastic processes: For, even though the original proof of that theorem used nonstandard analysis, the present paper contains a proof by means of classical measure theory (in the Appendix).

\section{A A "standard" proof of the consistency theorem for probabilistic regresses}

For all of this section, we fix two sequences $\left\langle\alpha_{k}\right\rangle_{k \in \mathbf{N}},\left\langle\beta_{k}\right\rangle_{k \in \mathbf{N}} \in[0,1]^{\mathbf{N}}$; as in all of this paper, $\mathbf{N}$ denotes the set of all nonnegative integers. In this section, we shall prove the following result, which is a slight generalisation of the consistency theorem in Herzberg [2010]:

Theorem A.1. There exists some probability measure $P$ and some sequence of $P$-measurable events $\left\langle S_{k}\right\rangle_{k \in \mathbf{N}}$ such that $P\left(S_{k} \mid S_{k+1}\right)=\alpha_{k}$ as well as $P\left(S_{k} \mid \complement S_{k+1}\right)=\beta_{k}$ for all $k \in \mathbf{N}$.

Essentially, the proof is based on the construction of a reversed nonhomogeneous Markov chain with state space $\{0,1\}$ that was started an infinitely long time ago. We use the notation $\prod_{\leftarrow}$ to refer to products of matrices whose order of multiplication has been reversed, so that $\prod_{k=m}^{n} T^{k}=T^{n} \cdots T^{m}$ for all $n \geq m \in \mathbf{N}$. Likewise, $\prod_{k=m}^{\infty} T^{k}=\lim _{n \rightarrow \infty} T^{n} \cdots T^{m}$.

proof by means of nonstandard analysis, cf. Herzberg [2012].

${ }^{6}$ To see this, apply the Transfer Principle to the sentence $\forall n \in \mathbf{N}(S(n+1) \Rightarrow \forall k \leq n(S(k)))$, which in turn follows from the hypothesis $\forall n \in \mathbf{N}(S(n+1) \Rightarrow S(n))$ by mathematical induction. 
For all $k \in \mathbf{N}$, put

$$
T^{k}=\left(\begin{array}{cc}
\alpha_{k} & 1-\alpha_{k} \\
\beta_{k} & 1-\beta_{k}
\end{array}\right) .
$$

For all $j, j^{\prime} \in \mathbf{N}$ with $j^{\prime} \geq j$ and for all $i, i^{\prime} \in\{0,1\}$, let

$$
Q_{i, i^{\prime}}^{j, j^{\prime}}={ }^{\top} e_{i^{\prime}}\left(\prod_{k=j}^{j^{\prime}-1} T^{k}\right) e_{i}
$$

wherein $e_{0}=\left(\begin{array}{l}0 \\ 1\end{array}\right)$ and $e_{1}=\left(\begin{array}{l}1 \\ 0\end{array}\right)$, and let

$$
Q_{i}^{j, \infty}={ }^{\top} c\left(\prod_{k=j}^{\infty} T^{k}\right) e_{i}
$$

for some $c=\left(\begin{array}{c}1-\varepsilon \\ \varepsilon\end{array}\right)$ with $\left.\varepsilon \in(0,1)\right]^{7}$ Define for all finite $J \subseteq \mathbf{N}$ with $\left\{j_{1}, \ldots, j_{\# J}\right\}$, a measure $P_{J}$ on $\{0,1\}^{J}$ by means of the equation

$$
P_{J}\{\vec{i}\}=\left(\prod_{\ell=1}^{\# J-1} Q_{i_{j_{\ell}, i_{j_{\ell}+1}}^{j_{\ell}, j_{\ell+1}}}\right) Q_{i_{j_{\# J} J}^{j_{\# J}, \infty}}^{\#}
$$

for all $\vec{i} \in\{0,1\}^{J}$.

Lemma A.2. $P_{J}$ is a probability measure on $\{0,1\}^{J}$ for all finite $J \subseteq \mathbf{N}$.

Proof. Note that all the $T^{k}$ are transition probability matrices, that the set of transition probability matrices is closed under multiplication and that the set of transition probability matrices is a closed subset of the set of matrices of that dimension. Therefore, $\prod_{\leftarrow}^{\leftarrow} T^{k}$ is a transition probability matrix. By definition, this means that each entry is nonnegative and the sum of each row vector equals 1. Formally,

$$
\left(\prod_{\overleftarrow{k}} T^{k}\right) \underbrace{\left(\begin{array}{c}
1 \\
1
\end{array}\right)}_{=e_{0}+e_{1}}=\left(\begin{array}{l}
1 \\
1
\end{array}\right),
$$

thus ${ }^{\top} e_{i}\left(\prod_{\leftarrow}^{\leftarrow} T^{k}\right)\left(e_{0}+e_{1}\right)=1$ for each $i \in\{0,1\}$. This immediately entails that

$$
Q_{0, i^{\prime}}^{j, j^{\prime}}+Q_{1, i^{\prime}}^{j, j^{\prime}}=1
$$

for all $j, j^{\prime} \in \mathbf{N}$ and each $i^{\prime} \in\{0,1\}$. However, it also means that

$$
\top^{\top} c\left(\prod_{\overleftarrow{k}} T^{k}\right)\left(e_{0}+e_{1}\right)=\left((1-\varepsilon)^{\top} e_{0}+\varepsilon^{\top} e_{1}\right)\left(\prod_{\overleftarrow{k}} T^{k}\right)\left(e_{0}+e_{1}\right)=1
$$

which implies that

$$
Q_{0}^{j, \infty}+Q_{1}^{j, \infty}=1
$$

${ }^{7}$ In the special case where $\varepsilon=\frac{1}{2}$, we have $Q_{i}^{j, \infty}=\frac{1}{2}$ for all $j \in \mathbf{N}$ and each $i \in\{0,1\}$. 
for all $j \in \mathbf{N}$. So,

$$
Q_{0, i^{\prime}}^{j, j^{\prime}}+Q_{1, i^{\prime}}^{j, j^{\prime}}=1, \quad Q_{0}^{j, \infty}+Q_{1}^{j, \infty}=1
$$

for all $j, j^{\prime} \in \mathbf{N}$ and each $i^{\prime} \in \mathbf{N}$. This can be used to prove inductively in $\# J$ that $\sum_{\vec{i} \in\{0,1\}^{J}} P_{J}\{\vec{i}\}=1$. Since clearly $P_{J}\{\vec{i}\} \geq 0$ for all $\vec{i} \in\{0,1\}^{J}$, we conclude that $P_{J}$ must be a probability measure on $\{0,1\}^{J}$.

Let $\mathcal{H}$ be the set of all finite subsets of $\mathbf{N}$.

Lemma A.3. $\left\langle P_{J}\right\rangle_{J \in \mathcal{H}}$ is a projective family of probability measures.

Proof. Let $H=J \cup\{h\}$ with $h \notin J$. Without loss of generality, let $h>\max J=$ $j_{\# J}$. Let $p_{J}^{H}$ be the projection of $H$ onto $J$. Then

$$
\begin{aligned}
& P_{H}\left(p_{J}^{H^{-1}}\{\vec{i}\}\right) \\
& =P_{H}(\{\vec{i}\} \times\{0,1\})=P_{H}(\{\vec{i}\} \times\{0\})+P_{H}(\{\vec{i}\} \times\{1\}) \\
& =\left(\prod_{\ell=1}^{\# J-1} Q_{i_{j_{\ell}}, i_{j_{\ell}+1}}^{j_{\ell}, j_{\ell+1}}\right) Q_{i_{j_{\# J}}, 0}^{j_{\# J}, h} Q_{0}^{h, \infty}+\left(\prod_{\ell=1}^{\# J-1} Q_{i_{j_{\ell}, j_{j_{\ell}+1}}^{j_{\ell}, j_{\ell+1}}}^{h,} Q_{i_{j_{\# J}}, 1}^{j_{\# J}, h} Q_{1}^{h, \infty}\right. \\
& =\left(\prod_{\ell=1}^{\# J-1} Q_{i_{j_{\ell}}, i_{j_{\ell}+1}}^{j_{,}, j_{\ell+1}}\right)\left(Q_{i_{j_{\# J}}, 0}^{j_{\# J}, h} Q_{0}^{h, \infty}+Q_{i_{j_{\# J}}, 1}^{j_{\# J}, h} Q_{1}^{h, \infty}\right)
\end{aligned}
$$

Now, it is not difficult to verify that

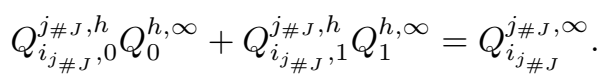

Indeed, $e_{0}{ }^{\top} e_{0}+e_{1}{ }^{\top} e_{1}=\left(\begin{array}{ll}1 & 0 \\ 0 & 1\end{array}\right)$, hence

$$
\begin{aligned}
& Q_{0}^{h, \infty} Q_{i_{j_{\# J},}^{j_{\# J}, h}}+Q_{1}^{h, \infty} Q_{i_{j_{\# J}}, 1}^{j_{\# J}, h} \\
& =\top^{\top} c\left(\prod_{k=h}^{\infty} T^{k}\right) e_{0}^{\top} e_{0}\left(\prod_{k=\overleftarrow{j_{\# J}}}^{h-1} T^{k}\right) e_{i_{j_{\# J}}} \\
& +{ }^{\top} c\left(\prod_{k=h}^{\infty} T^{k}\right) e_{1}{ }^{\top} e_{1}\left(\prod_{k=j_{\# J}}^{h-1} T^{k}\right) e_{i_{j_{\# J}}} \\
& =\top^{\top}\left(\prod_{k=j_{\# J}}^{h-1} T^{k}\right)\left(\prod_{k=h}^{\infty} T^{k}\right) e_{i_{j_{\# J} J}}={ }^{\top} c\left(\prod_{k=j_{\# J}}^{\infty} T^{k}\right) e_{i_{j_{\# J}}}^{\infty}=Q_{i_{j_{\# J} J}^{j}, \infty} .
\end{aligned}
$$

Inserting Equation (5) into Equation (4) yields

$$
P_{H}\left(p_{J}^{H^{-1}}\{\vec{i}\}\right)=\left(\prod_{\ell=1}^{\# J-1} Q_{i_{j_{\ell}}, i_{j_{\ell}+1}}^{j_{\ell}, j_{\ell+1}}\right) Q_{i_{j_{\# J}}^{j_{\# J}}, \infty}^{m}
$$


whence by definition $P_{H}\left(p_{J}^{H^{-1}}\{\vec{i}\}\right)=P_{J}\{\vec{i}\}$ for arbitrary $\vec{i} \in\{0,1\}^{J}$ and all finite $H, J \subseteq \mathbf{N}$ with $J \subseteq H$ and $\#(H \backslash J)=1$. This implies (cf. Bauer $\left[2002\right.$, p. 307, Bemerkung 2]) that $\left\langle P_{J}\right\rangle_{J \in \mathcal{H}}$ is a projective family of probability measures.

In the following theorem, $\mathcal{P}(\{0,1\})^{\otimes \mathbf{N}}$ denotes the infinite product of countably many identical copies of the power-set of $\{0,1\}$; it is the smallest $\sigma$-algebra with respect to which all projections are measurable. Given any $J \in \mathcal{H}$, the map $\pi_{J}$ is the projection onto $\{0,1\}^{J}$ defined as the map $\pi_{J}:\{0,1\}^{\mathbf{N}} \rightarrow\{0,1\}^{J}, \quad \omega \mapsto\langle\omega(k)\rangle_{k \in J}$

Theorem A.4. There exists a probability measure $P: \mathcal{P}(\{0,1\})^{\otimes \mathbf{N}} \rightarrow[0,1]$ such that $P_{J}=P\left(\pi_{J}^{-1}(\cdot)\right)$ for all $J \in \mathcal{H}$.

Proof. By the Daniell-Kolmogorov theorem, there must be a probability measure $P$ on $\Omega=\{0,1\}^{\mathbf{N}}$ such that the finite-dimensional distributions of the process $X$ defined by $X_{k}: \Omega \rightarrow\{0,1\}, \quad \omega \mapsto \omega(k)$ (for all $k \in \mathbf{N}$ ) coincide with the probability measures $P_{J}, J \in \mathcal{H}$.

Now we can, at last, prove the consistency theorem stated at the beginning as a corollary to Theorem A.4.

Proof of Theorem A.1. Let $P$ and $X$ be as in the proof of Theorem A.4. For all $k \in \mathbf{N}$, set $S_{k}=\left\{X_{k}=1\right\}$, so that $\complement S_{k}=\left\{X_{k}=0\right\}$,

Then, for all $n>k \in \mathbf{N}$,

$$
P\left\{X_{k}=1\right\}=T^{k} \cdots T^{n}\left(\begin{array}{c}
P\left\{X_{n+1}=1\right\} \\
P\left\{X_{n+1}=0\right\}
\end{array}\right),
$$

hence

$$
P\left(S_{k}\right)=T^{k} \cdots T^{n}\left(\begin{array}{c}
P\left(S_{n+1}\right) \\
1-P\left(S_{n+1}\right)
\end{array}\right)
$$

as well as

$$
T^{k}=\left(\begin{array}{ll}
P\left(\left\{X_{k}=1\right\} \mid\left\{X_{k+1}=1\right\}\right) & P\left(\left\{X_{k}=0\right\} \mid\left\{X_{k+1}=1\right\}\right) \\
P\left(\left\{X_{k}=1\right\} \mid\left\{X_{k+1}=0\right\}\right) & P\left(\left\{X_{k}=0\right\} \mid\left\{X_{k+1}=0\right\}\right)
\end{array}\right),
$$

whence

$$
T^{k}=\left(\begin{array}{cc}
P\left(S_{k} \mid S_{k+1}\right) & P\left(\complement S_{k} \mid S_{k+1}\right) \\
P\left(S_{k} \mid \complement S_{k+1}\right) & P\left(\complement S_{k} \mid \complement S_{k+1}\right)
\end{array}\right) .
$$

Comparing this with the definition of $T^{k}$ in Equation (3) leads us to conclude

$$
P\left(S_{k} \mid S_{k+1}\right)=\alpha_{k}, \quad P\left(S_{k} \mid \complement S_{k+1}\right)=\beta_{k}
$$

for all $k \in \mathbf{N}$. 


\section{References}

Atkinson, D. and Peijnenburg, J. (2010). The solvability of probabilistic regresses. A reply to Frederik Herzberg. Studia Logica, 94(3):347-353.

Bauer, H. (2002). Wahrscheinlichkeitstheorie. 5., durchgesehene und verbesserte Auflage. de Gruyter, Berlin.

Fumerton, R. (1998). Replies to my three critics. Philosophy and Phenomenological Research, 58:927-937.

Fumerton, R. (2001). Epistemic justification and normativity. In Steup, M., editor, Knowledge, truth, and duty: Essays on epistemic justification, responsibility and virtue, pages 49-61. Oxford University Press, New York.

Gillett, C. (2003). Infinitism redux? A response to Klein. Philosophy and Phenomenological Research, 66:709-717.

Herzberg, F. (2008a). A definable nonstandard enlargement. Mathematical Logic Quarterly, 54(2):167-175.

Herzberg, F. (2008b). Addendum to "A definable nonstandard enlargement". Mathematical Logic Quarterly, 54(6):666-667.

Herzberg, F. (2010). The consistency of probabilistic regresses. A reply to Jeanne Peijnenburg and David Atkinson. Studia Logica, 94(3):331-345.

Herzberg, F. (2012). Simple nonstandard proofs of Daniell-Kolmogorov-type theorems. Positivity, 16(4):627-631.

Jech, T. (2000). Set theory. The third millennium edition. Springer Monographs in Mathematics. Springer, Berlin.

Kanovei, V. and Reeken, M. (2004). Nonstandard analysis, axiomatically. Springer Monographs in Mathematics. Springer, Berlin.

Kanovei, V. and Shelah, S. (2004). A definable nonstandard model of the reals. Journal of Symbolic Logic, 69(1):159-164.

Klein, P. (1998). Foundationalism and the infinite regress of reasons. Philosophy and Phenomenological Research, 58:919-925.

Klein, P. (1999). Human knowledge and the infinite regress of reasons. Philosophical Perspectives, pages 297-325.

Klein, P. (2005). Infinitism is the solution to the regress problem. In Steup, M. and Ernest, S., editors, Contemporary debates in epistemology, pages 131-140. Blackwell, Malden, M.A.

Klein, P. (2007a). How to be an infinitist about doxastic justification. Philosophical Studies, 134:25-29.

Klein, P. (2007b). Human knowledge and the infinite progress of reasoning. Philosophical Studies, 134:1-17. 
Loeb, P. (1975). Conversion from nonstandard to standard measure spaces and applications in probability theory. Transactions of the American Mathematical Society, 211:113-122.

Nelson, E. (1977). Internal set theory: A new approach to nonstandard analysis. Bulletin of the American Mathematical Society, 83(6):1165-1198.

Peijnenburg, J. (2007). Infinitism regained. Mind, 116(463):597-602.

Peijnenburg, J. (2010). Ineffectual foundations: Reply to Gwiazda. Mind, 119(476):1125-1133.

Peijnenburg, J. and Atkinson, D. (2008). Probabilistic justification and the regress problem. Studia Logica, 89(3):333-341.

Peijnenburg, J. and Atkinson, D. (2011). Grounds and limits: Reichenbach and foundationalist epistemology. Synthese, 181(1):113-124.

Podlaskowski, A. and Smith, J. (2011). Infinitism and epistemic normativity. Synthese, 178:515-527.

Schuster, P., Berger, U., and Osswald, H., editors (2001). Reuniting the antipodes - constructive and nonstandard views of the continuum, volume 306 of Synthese Library, Dordrecht. Kluwer Academic Publishers.

Turri, J. (2009). On the regress argument for infinitism. Synthese, 166:157-163. 